\title{
Less surgical site infections in neurosurgery during COVID-19 times-one potential benefit of the pandemic?
}

\author{
T. Chacón-Quesada ${ }^{1} \cdot$ V. Rohde ${ }^{1} \cdot$ C. von der Brelie ${ }^{1}$
}

Received: 2 January 2021 / Revised: 8 February 2021 / Accepted: 22 February 2021 / Published online: 5 March 2021

(C) The Author(s) 2021

\begin{abstract}
Hygiene measures were intensified when the COVID-19 pandemic began. Patient contacts were limited to a minimum. Visitors were either not allowed for a certain period or limited for the rest of the time. The hospital staff began to wear masks and gloves continuously. Clinical examinations and routine wound controls were also performed under intensified hygiene standards. These circumstances result in a limitation of direct physical interactions between the nursing staff, the physicians and the patients. We analyzed to what extent the intensification of hygiene measures affects the rate of surgical site infections (SSI) after neurosurgical procedures. The rate of SSI during the 6-month interval after the beginning of COVID-19 measures was compared with the SSI rate before. The numbers of the period before COVID-19 were analyzed as mean values resulting from the analysis of two separate time periods each consisting of 6 months. The spectrum of surgical procedures was compared. Patient-related risk factors for SSIs were noted. Microorganisms were analyzed. We focused on SSIs occurring at a maximum of 60 days after the primary surgery. Overall, in the two respective 6-month periods before COVID-19, a mean of 1379 patients was surgically treated in our institution. After the beginning of COVID-19 (starting from 04/2020) our surgical numbers dropped by 101, resulting in a total number of 1278 patients being operated after 03/2020 until 09/2020. The SSI rate was 3.6\% (03/2019-09/2019, 50 SSIs) and 2.2\% (09/2019-03/2020, 29 SSIs), resulting in a mean of 2.9\% before COVID-19 began. After the beginning of COVID-19 hygiene measures, this rate dropped to $1.4 \%$ (16 SSIs) resembling a significant reduction $(p=0.003)$. Risk factors for the development of SSI were present in $81.3 \%$ of all patients. Pre- and post-COVID-19 patient groups had similar baseline characteristics. The same holds true when comparing the percentage of cranial and spinal procedures pre- and post-COVID-19 $(p=0.91)$. Comparing the numbers $(p=0.28)$ and the species $(p=0.85)$ of microorganisms (MO) causing SSI, we found a similar distribution. Despite equal demographics and characteristics of SSI, the rate of SSI dropped substantially. This argues for an effective reduction of postoperative SSI resulting from the implementation of strict hygiene measures being established after the beginning of the COVID-19 pandemic. We therefore advocate continuing with strict and intensive hygiene measures in the future.
\end{abstract}

Keywords Neurosurgery $\cdot$ COVID-19 $\cdot$ Pandemic $\cdot$ Surgical site infection

\section{Introduction}

The COVID-19 pandemic has affected either directly or indirectly all medical fields. It has also exercised a huge influence on neurosurgery. The admission rate of patients was significantly affected [7]. Furthermore, certain types of elective neurosurgical procedures like transnasal surgeries, which were

C. von der Brelie

cvdb@gmx.net

1 Department of Neurosurgery, Georg August University of Göttingen, Göttingen, Germany considered to be high-risk procedures for Sars-Cov2 transmission, had to be cancelled unless the surgery had to be indicated because of a neurological deficit. Despite the transmission and infection risks, neurosurgical procedures were performed in large numbers also during the pandemic. Sadly, our devoted neurosurgical care leads to the fact that some of our neurosurgical colleagues died due to the pandemic [1].

Prohibition of virus transmission is a key factor when it comes to measures curtailing the virus spread and "flattening the curve." Thus, according to the local institutional guidelines, hygiene and public health measures have been significantly intensified to both protect patients and health workers in every health care institution. The beginning of these 
hygiene protocol changes was in March 2020 in Germany. Visitors were either not allowed for a certain period or limited for the rest of the time. The hospital staff began to wear masks and gloves continuously. Clinical examinations and routine wound controls were also performed under intensified hygiene standards. These circumstances resulted in a limitation of direct physical interactions between the nursing staff, physicians and patients. Postoperative surgical site infections (SSI) following cranial and spinal surgery are dreaded complications affecting the postoperative outcome, influencing morbidity and mortality and significantly reduce the patients' life quality [4, 9-11]. Prevalences of SSIs in neurosurgery range from $0.5 \%$ in elective cranial or spinal surgery up to $20 \%$ or more in revision cases or after open or penetrating traumatic brain injury $[2,5]$.

The goal of the present study was to analyze the rate of SSIs after the beginning of COVID-19 hygiene measures.

\section{Methods}

We performed a retrospective observational analysis of all SSIs treated in our department in adult patients since the instauration of the hygiene measurements related to the lockdown due to the COVID-19 pandemic from April until the end of September 2020. This analysis was carried out respecting the guidelines of good clinical practice following institutional ethical guidelines.

The main changes of the hygiene protocol are highlighted in Table 1.

Infections treated 12 months prior were also analyzed and divided into two groups both resembling 6-month time intervals (March 2019-August 2019, September 2019-February 2020). The data of the period before COVID-19 was analyzed as mean values resulting from the examination of these two separate time periods. Clinical data and demographics were recollected in order to compare not only infection rates but also clinical characteristics among the groups. For this part of the analysis, SSIs that developed more than 60 days after the initial surgery were excluded to focus on infections related to

Table 1 Key hygiene measures implemented in our institution postoperative wound care and the implementation of COVID19 hygiene measures by the hospital staff and exclude possible cofounders or causes out of the scope of our research. Wound revisions after surgeries being performed in other hospitals were not included. In purpose to reduce a potential confounding factor resulting from intrinsically infectious pathologies (brain abscesses, spondylodiscitis, other primary infectious diseases or surgery for open-traumatic brain injury), all of those cases are excluded, no matter if they were performed at our institution or not.

Patient demographics included age, sex and the preexistence of patient-related risk factors for SSI. Patientrelated risk factors considered were obesity, smoking, diabetes mellitus and vascular diseases. Other known risk factors analyzed were history of multiple interventions in the same surgical site, radiation and cerebrospinal fluid leak after the initial surgery. Numbers, multiplicity and type of pathogenic microorganisms (MOs) were analyzed. Surgeries were categorized in spinal or cranial procedures. Infections were classified in deep or superficial according to bone involvement. Information regarding an initial conservative treatment through antibiotic therapy was also recollected.

SPSS software (IBM Corp., Armonk, NY, USA) was used for statistical analysis. Continuous variables are presented as mean values and standard deviation. Categorical variables are depicted as numbers and percentages each. Categorical variables were compared in a cross-table fashion; statistical differences for categorical variables were calculated using chisquare test. After testing for normality, we applied a $t$ test for statistical significance for continuous variables. A $p$ value of $<$ 0.05 was considered to represent significant differences.

\section{Results}

Overall, in the first respective 6-months period before COVID19,1377 patients were surgically treated in our institution. In the second time interval, 1381 patients underwent surgery, resulting in a mean of 1379 patients. After the instauration of the COVID19 measures, our surgical numbers decreased by 101 . The SSI rate was 3.6\% (03/2019-09/2019, 50 SSIs) and 2.2\% (09/201903/2020, 29 SSIs), resulting in a mean of $2.9 \%$ before COVID19 began. After the beginning of COVID-19 hygiene measures, this rate dropped to $1.4 \%$ (16 SSIs). This resembles a significant reduction of SSI rates $(p=0.003)$.

Risk factors for the development of SSI were present in $81.3 \%$ of all patients. Comparison of pre- and post-COVID-19 groups revealed no differences (see Table 1). The site of SSIs did not differ regarding cranial and spinal procedures $(p=0.91)$.

Comparing the number $(p=0.28)$ and the species $(p=0.85)$ of microorganisms (MO) causing SSI, we found a similar distribution between the cohorts pre- and post-COVID-19 (see Tables 2 and 3). 
Table 2 Clinical and demographic characteristics of patients with SSI

\begin{tabular}{lllll}
\hline Factor & & Before COVID- & $\begin{array}{l}\text { During COVID- } \\
19\end{array}$ & $\begin{array}{l}p \\
\text { value }\end{array}$ \\
\hline Age (mean, SD) & & 19 & $62(15.2)$ & 0.44 \\
Sex & M & $29(49.2 \%)$ & $8(50 \%)$ & N/A \\
Type of procedure & F & $30(50.2 \%)$ & $8(50 \%)$ & \\
& Cranial & $36(61 \%)$ & $10(62.5 \%)$ & 0.91 \\
Preexisting patient-related risk factors & Spinal & $23(39 \%)$ & $6(37.5 \%)$ & \\
& Yes & $47(79.7 \%)$ & $14(87.5 \%)$ & 0.48 \\
Time span to onset of SSI (SD) & No & $12(20.3 \%)$ & $2(12.5 \%)$ & \\
Multiple surgeries? & & $30(14.3)$ & $27(13.1)$ & 0.45 \\
& Yes & $11(18.6 \%)$ & $5(31.2 \%)$ & 0.27 \\
Post surgical radiation & No & $48(81.4 \%)$ & $11(68.8 \%)$ & \\
& Yes & $4(6.8 \%)$ & $2(12.5 \%)$ & 0.45 \\
Post surgical CSF leakage & No & $55(93.2 \%)$ & $14(87.5 \%)$ & \\
& Yes & $3(5.1 \%)$ & $2(12.5 \%)$ & 0,29 \\
Pre-revision conservative antibiotic & No & $56(94.9 \%)$ & $14(87.5 \%)$ & \\
therapy & Yes & $15(25.4 \%)$ & $2(12.5 \%)$ & 0.23 \\
Type of infection & No & $44(74.6 \%)$ & $14(87.5 \%)$ & \\
& Superficial & $20(33.9 \%)$ & $8(50 \%)$ & 0.24 \\
& Deep & $39(66.1 \%)$ & $8(50 \%)$ & \\
\hline
\end{tabular}

\section{Discussion}

\section{Main results}

Our analysis showed that the rate of SSI dropped dramatically since the beginning of the COVID-19 pandemic. This result was highly significant. In general, we were not surprised by the result that the rate of SSIs dropped since it seems logical that an increase in hygiene measures would affect the rate of postoperative SSIs, but the amplitude of the change is dramatic. No other measure in the last decades was able to create such a dramatic drop in SSI rate.

Overall numbers of surgical procedures only changed a little. Nonetheless, the numbers of procedures with a relatively low risk of SSIs (facet joint infiltrations, thermocoagulations of facet joint or iliosacral joint) were even performed less often after COVID-19. Moreover, the time interval after the beginning of the pandemic contains the warmer months of the year. Still, SSIs are considered to be higher in the warmer months of the year [3]. Both of these aspects result in a relatively (potentially false) high SSI rate after COVID-19 in our study.

The patients in the pre-COVID-19 era were comparable to the patients who underwent surgery during the COVID-19 pandemic. Risk factors for SSI development were also equally distributed among the groups. We also did not expect a change in the pathogenic MOs resulting in SSI. Our data confirmed this expectation. Most of the general anesthetic and surgical procedures have not changed. The patients still underwent the basic SSIprohibitive protocols like preoperative antiseptic skin cleaning, preoperative antibiotics, glycemic control, postoperative wound checks and eventual postoperative antibiotic treatment.
Nonetheless, as in many other institutions, our hygiene measures intensified in every different part of in-patient care as part of the public health response to the pandemic. The main aspects are full-protective personal equipment of all personnel in the OR. Induction of anesthesia was oftentimes done with the minimum personnel since in many surgeries the COVID-19 infection status was not completely worked up. Also, intermediate cleaning procedures in the OR were intensified. The staff being involved in the pre- and postoperative treatment also continuously wore masks, oftentimes gowns and gloves. Patients themselves were also aware of the importance of hand hygiene, masks and social distancing. Wound checks were regularly performed, but with full-protective equipment and most of the times by only one physician. To sum it up, physical contact between care personnel and the patients has been reduced to an absolute minimum. This also holds true for physicians, ergo- and speech-therapists as well as psychologists. In general, but with few exceptions, visitors were not allowed since the pandemic started. This de-facto isolation of the patients potentially also contributes to the drop in the SSI rate.

\section{Generalizability}

As expected, other studies have shown similar results. Hussain et al. in a recent article have shown similar results in a cohort study including patients who underwent thoracic surgery [6]. The same trend was shown by Losurdo and colleagues, who reported a drop from 8.4 to $3.3 \%$ in a cohort of patients who were operated in a general surgical department [8]. 
Table 3 Microbiology

\begin{tabular}{|c|c|c|c|c|c|c|c|c|}
\hline Factor & & Before COVID-19 & Cranial & Spinal & During COVID-19 & Cranial & Spinal & $p$ value \\
\hline \multirow[t]{3}{*}{ Single or multiple MO? } & No MO detected & $13(22.0 \%)$ & 5 & 8 & $5(29.4 \%)$ & 1 & 4 & \multirow[t]{3}{*}{0.38} \\
\hline & Single MO & $32(54.2 \%)$ & 21 & 11 & $6(35.3 \%)$ & 4 & 2 & \\
\hline & Multiple MOs & $14(23.7 \%)$ & 10 & 4 & $6(35.3 \%)$ & 5 & 1 & \\
\hline \multirow[t]{3}{*}{ Detected pathogens } & Staphylococci & 39 & 26 & 13 & 10 & 7 & 3 & \multirow[t]{3}{*}{0.85} \\
\hline & Other Gram-positive bacteria & 11 & 8 & 3 & 4 & 4 & 0 & \\
\hline & Gram-negative Bacteria & 12 & 9 & 3 & 4 & 3 & 1 & \\
\hline
\end{tabular}

\section{Limitations}

Given our data, we can describe just an association between the COVID-19 hygiene measures. We can just speculate that the dramatic reduction in SSI is to be explained with the change of hygiene measures; nonetheless, this conclusion seems rational. Furthermore, we could not determine which part of the intensified hygiene measures is the most important change, although this would be very interesting in order to understand the impact of the different parts of the hygienic strategy. Our analysis only covers a 6-month period, which, as a result, may lead to an underrepresentation of the SSI rate.

\section{Conclusions}

Despite equal demographics and characteristics of SSI, the rate of SSI dropped dramatically. This argues for an effective reduction of postoperative SSI resulting from the implementation of strict hygiene measures being established after the beginning of the COVID-19 pandemic. We therefore advocated continuing with the strict hygiene measures in the future.

Author contribution Tatiana Chacon extracted the data from the hospital files, analyzed the data, performed the statistic and drafted the manuscript. Veit Rohde drafted the manuscript. Christian von der Brelie gave the idea, analyzed the data, performed the statistic and drafted the manuscript.

Funding Open Access funding enabled and organized by Projekt DEAL.

Data Availability The datasets analyzed during the current study are available from the corresponding author on reasonable request. All data generated or analyzed during this study are included in this published article.

Code availability Not applicable

\section{Declarations}

Ethics approval The study was carried out respecting the guidelines of good clinical practice. Ethical approval was waived since.
Consent to participate Not applicable since this was a retrospective data analysis.

Consent for publication Not applicable since this was a retrospective analysis.

Conflict of interest The authors declare no competing interests.

Open Access This article is licensed under a Creative Commons Attribution 4.0 International License, which permits use, sharing, adaptation, distribution and reproduction in any medium or format, as long as you give appropriate credit to the original author(s) and the source, provide a link to the Creative Commons licence, and indicate if changes were made. The images or other third party material in this article are included in the article's Creative Commons licence, unless indicated otherwise in a credit line to the material. If material is not included in the article's Creative Commons licence and your intended use is not permitted by statutory regulation or exceeds the permitted use, you will need to obtain permission directly from the copyright holder. To view a copy of this licence, visit http://creativecommons.org/licenses/by/4.0/.

\section{References}

1. Chaurasia B, Deora H, El-Ghandour NMF, Oyesiku NM, Chaurasia RK, Schulder M, Sanchez JAS, Teo M, Hernesniemi J, Linzey JR, Schwartz TH, Cohen-Gadol AA, Lawton M, Umana G, Mura J, Grotenhuis A, Sinha AK, Schroeder HWS, Natarajan S, Sughrue ME, Spetzler RF, Drummond K, Tanikawa R, Do Seixo Kadri PA, Kato Y, Teo C, Suri A, Tomasi SO, Winkler PA, Scalia G, Sampron N, Rasulic L, Cappabianca P, Fontanella MM, Laws ER (2020) In memoriam: A memoir for our fallen "heroes.". Neurosurgery 87:854-856. https://doi.org/10.1093/neuros/ nyaa314

2. Crocker A, Kornilo A, Conly J, Henderson E, Rennert-May E, Leal $\mathrm{J}$ (2020) Using administrative data to determine rates of surgical site infections following spinal fusion and laminectomy procedures. Am J Infect Control. https://doi.org/10.1016/j.ajic.2020.10.010

3. Durkin MJ, Dicks KV, Baker AW, Moehring RW, Chen LF, Sexton DJ, Lewis SS, Anderson DJ (2015) Postoperative infection in spine surgery: does the month matter? J Neurosurg Spine 23: 128-134. https://doi.org/10.3171/2014.10.SPINE14559

4. Farah K, Lubiato A, Meyer M, Prost S, Ognard J, Blondel B, Fuentes S (2020) Surgical site infection following surgery for spinal deformity: About 102 patients. Neurochirurgie. https://doi.org/10. 1016/j.neuchi.2020.10.006

5. Harrington BM, Gretschel A, Lombard C, Lonser RR, Vlok AJ (2020) Complications, outcomes, and management strategies of 
non-missile penetrating head injuries. J Neurosurg:1-9. https://doi. org/10.3171/2020.4.jns20122

6. Hussain A, Ike DI, Durand-Hill M, Ibrahim S, Roberts N (2020) Sternal wound infections during the COVID-19 pandemic: an unexpected benefit. Asian Cardiovasc Thorac Ann 021849232097763. https://doi.org/10.1177/0218492320977633

7. Krenzlin H, Bettag C, Rohde V, Ringel F, Keric N (2020) Involuntary ambulatory triage during the COVID-19 pandemic A neurosurgical perspective. PLoS One 15(6):e0234956. https:// doi.org/10.1371/journal.pone.0234956

8. Losurdo P, Paiano L, Samardzic N, Germani P, Bernardi L, Borelli M, Pozzetto B, de Manzini N, Bortul M (2020) Impact of lockdown for SARS-CoV-2 (COVID-19) on surgical site infection rates: a monocentric observational cohort study. Updat Surg 72. https:// doi.org/10.1007/S13304-020-00884-6

9. Rotter J, Dowlati E, Cobourn K, Kalhorn C (2020) A crosssectional analysis of variables associated with morbidity and mortality in postoperative neurosurgical patients diagnosed with sepsis. Acta Neurochir 162:2837-2848. https://doi.org/10.1007/ s00701-020-04586-1

10. Vinas-Rios JM, Rauschmann M, Sellei R, Arabmotlagh M, Sobotke R, Meyer F (2020) Intra-and postoperative complications in the treatment of spinal metastases. A multicentre surveillance study from the German Spinal Registry (DWG-Register). J Neurosurg Sci. https://doi.org/10.23736/S0390-5616.20.05029-8

11. Weber WP, Zwahlen M, Reck S, Feder-Mengus C, Misteli H, Rosenthal R, Brandenberger D, Oertli D, Widmer AF, Marti WR (2008) Economic Burden of Surgical Site Infections at a European University Hospital. Infect Control Hosp Epidemiol 29:623-629. https://doi.org/10.1086/589331

Publisher's note Springer Nature remains neutral with regard to jurisdictional claims in published maps and institutional affiliations. 